\title{
RESÍDUOS ORGÂNICOS COMO RECONDICIONANTE DE SUBSOLO DEGRADADO E EFEITOS NA ATIVIDADE MICROBIANA E FERTILIDADE EM CULTIVO DE BARBATIMÃO'
}

\author{
Hemerson Fernandes Calgaro ${ }^{2}$, Ana Maria Rodrigues Cassiolato ${ }^{3}$, Walter Veriano Valério Filho ${ }^{4}$, Francisco
} Maximino Fernandes ${ }^{3}$ e Kátia Luciene Maltoni ${ }^{3}$

\begin{abstract}
RESUMO - Com a construção da Usina Hidrelétrica de Ilha Solteira no final da década de 1960, algumas áreas foram desmatadas, servindo como "área de empréstimo". O solo dessas áreas foi extraído, e estas adquiriram características químicas e biológicas distantes das ideais. $\mathrm{O}$ trabalho objetivou avaliar o uso de resíduos orgânicos como recondicionante de subsolo degradado e quantificar os efeitos na atividade microbiana e na fertilidade de solo cultivado com barbatimão (Stryphnodendron polyphyllum Mart.). A área localiza-se na fazenda da UNESP/Campus de Ilha Solteira, em Selvíria, MS, onde foram alocados quatro blocos (repetições) de $250 \mathrm{~m}^{2}$ $(10 \times 25 \mathrm{~m})$ com 10 tratamentos cada, sendo testemunha; calagem; adubação com N+P; calagem +N+P; N+P+aguapé; $\mathrm{N}+\mathrm{P}+$ bagaço de cana; $\mathrm{N}+\mathrm{P}+$ aguapé+bagaço de cana; calagem $+\mathrm{N}+\mathrm{P}+$ aguapé; calagem $+\mathrm{N}+\mathrm{P}+$ bagaço de cana e calagem $+\mathrm{N}+\mathrm{P}+$ aguapé+bagaço de cana. Avaliaram-se as características químicas do subsolo, crescimento da planta, carbono da biomassa microbiana $(\mathrm{CBM})$ e do $\mathrm{C}-\mathrm{CO}_{2}$ liberado, quociente metabólico $\left(q \mathrm{CO}_{2}\right)$ e $(q \mathrm{MIC})$, em cinco épocas de amostragens de subsolo (junho, agosto, novembro e dezembro de 2005 e março de 2006). O subsolo continua a apresentar caráter ácido e pobre em nutrientes, após um ano de avaliação. Já o carbono da biomassa mostrou tendência à estabilização, enquanto o $q \mathrm{CO}_{2}$ e o quociente microbiano $q \mathrm{MIC}$ diminuíram no mesmo período. No conjunto, embora longe do ideal, os tratamentos com resíduos orgânicos apresentaram melhores resultados, com ligeiro diferencial positivo para a presença de aguapé. O CBM permitiu a observação de variação sazonal.
\end{abstract}

Palavras-chave: Subsolo cerrado, aguapé e bagaço de cana-de-açúcar.

\section{ORGANIC RESIDUES USED AS DEGRADED SUBSOIL RECONDITIONER ND THEIR EFFECT ON SOIL MICROBIAL ACTIVITY AND SOIL FERTILITY IN BARBATIMÃO CULTIVATION}

\begin{abstract}
Following the construction of the Hydroelectric Power Station of Solteira Island in the late 1960 s, some areas were deforested and used as "lending areas". The soil was extracted from these areas, which acquired chemical and biological characteristics far from the ideal. This work aimed to evaluate the use of organic residues as a reconditioner of degraded subsoil and quantify its effects on soil microbial activity and fertility in 'barbatimão'(Stryphnodendron polyphyllum Mart.) cultivation. The area is located at the UNESP/Ilha Solteira Campus farm, in Selvíria - MS. Four sections (repetitions) of $250 \mathrm{~m}^{2}(10 \times 25 \mathrm{~m})$ were allocated, with 10 treatments for each, as follows: control; liming; fertilizing $+N+P$; liming $+N+P ; N+P+$ water hyacinth; $N+P+$ sugar cane bagasse; $N+P+$ water hyacinth + sugar cane bagasse; liming $+N+P+$ water hyacinth;
\end{abstract}

\footnotetext{
${ }^{1}$ Recebido em 20.06.2007 e aceito para publicação em 22.08.2008.

${ }^{2}$ ProgramadePós-GraduaçãoemAgronomiadaUniversidadeEstadual PaulistaJúliodeMesquitaFilho(UNESP).E-mail:〈hemerfc@yahoo.com.br>

${ }^{3}$ Departamento de Fitossanidade Engenharia Rural e Solos da UNESP. E-mail: <anamaria@ bio.feis.unesp.br>.

${ }^{4}$ Departamento de Matemática da UNESP. E-mail: <wvvf@fqm.feis.unesp.br>.
} 
liming $+N+P+$ sugar cane bagasse and liming $+N+P+$ water hyacinth + sugar cane bagasse. The chemical characteristics of the subsoil were evaluated as well as plant growth, carbon microbial biomass (CMB) and released C$\mathrm{CO}_{2}$, metabolic quotient $\left(q \mathrm{CO}_{2}\right)$ and microbial quotient (qMIC), in 5 subsoil sample collecting times (June, August, November and December, 2005 and March, 2006). The subsoil displayed the same acid and poor nutrient level characteristics after a year. During the experiment, the CMB presented stabilization tendencies and the $\mathrm{qCO} 2$ and $\mathrm{q} M I C$ decreased in the same period. In all, even below the ideal level, the organic residues showed better results, with small positive differences for water hyacinth. CMB exhibited seasonal variations.

Keywords: Subsoil, savannah, water hyacinth and sugar cane bagasse.

\section{INTRODUÇÃO}

Com a construção da Usina Hidrelétrica de Ilha Solteira, no Município de Selvíria, MS, surgiram áreas degradadas, como as "áreas de empréstimo", cuja caracterização química mostrou que o subsolo é pobre em nutrientes e matéria orgânica, além de propriedades físicas comprometidas e distantes das condições naturais, para o bioma Cerrado (RODRIGUES et al., 2007).

O uso da cobertura vegetal como medida mitigadora dos impactos ambientais é uma opção prática, econômica e coerente para a recuperação de áreas degradadas (D'ALTERIO e VALCARCEL, 1996). O barbatimão (Stryphnodendron polyphyllum Mart.), leguminosa pertencente à família Fabaceae, ademais de seu uso medicinal, tem sido recomendada para composição de reflorestamentos mistos, visando à recuperação da vegetação de áreas degradadas e de preservação permanente (LORENZI, 2002; SOUZA e LORENZI, 2005).

Os índices microbiológicos podem ser capazes de discriminar com maior rapidez do que os parâmetros físico-químicos (CATTELAN e VIDOR, 1992), os efeitos de diferentes sistemas de manejo sobre a qualidade do solo (PAPENDIK et al., 1992). Nesse sentido, a quantificação do carbono da biomassa microbiana (CBM) representa a fração ativa e biodegradável da matéria orgânica e reflete as tendências de mudanças que estão ocorrendo nela, no médio e longo prazos (FEIGL et al., 1998). A respiração microbiana, estimada por meio do carbono do $\mathrm{CO}_{2}\left(\mathrm{C}-\mathrm{CO}_{2}\right)$ liberado, possibilita verificar a velocidade com que ocorre a liberação dos nutrientes para as plantas, bem como as perdas de carbono orgânico total do solo (D’ANDRÉA et al., 2002).

O quociente metabólico $\left(q \mathrm{CO}_{2}\right)$, relação entre C$\mathrm{CO}_{2} / \mathrm{CBM}$, é um indicador de estresse da biomassa microbiana, pois expressa a eficiência com que a biomassa microbiana imobiliza carbono (MONTEIRO e GAMARODRIGUES, 2004). O quociente microbiano ( $q \mathrm{Cmic}$ ), ou seja, a porcentagem do CBM em relação ao carbono orgânico (Corg) do solo, possibilita acompanhar, de forma mais rápida, as perturbações sofridas pelo desequilíbrio ecológico e as variações no total de matéria orgânica (CATTELAN e VIDOR, 1992).

O aumento da fertilidade do solo, por meio da adubação química e orgânica, pode provocar alterações no solo, ajudando a sua recuperação. Nesse sentido, o aguapé (Eichornia crassipes (Mart.) Solms.) tem mostrado potencial. É uma planta monocotiledônea, herbácea, classificada como aquática ou macrófita flutuante, perene e nativa do Brasil (BEYRUTH, 1992), que apresenta aumento de área de $15 \%$ ao dia, produzindo 480 t ha $^{-1}$ ano $^{-1}$ de massa verde (LORENZI, 1991; MARCONDES e TANAKA, 1997). Sua fitomassa, rica em macro e micronutrientes, é excelente para solos erodidos, exauridos, desestruturados, desprovidos de húmus, matéria orgânica e, conseqüentemente, sem vida (LUTZENBERGER, 1985).

A cana-de-açúcar (Saccharum sp.) é uma planta considerada detentora dos mecanismos fisiológicos mais aperfeiçoados, eficiência de assimilação de fotossíntese e capacidade de produzir massa verde (ULLER et al., 1999). Como subproduto da fabricação de açúcar e do álcool, o bagaço vem sendo utilizado como matéria-prima para geração de energia elétrica, alimentação animal e componente no processo de compostagem (PARANHOS, 1987), além de apresentar potencial para melhorar as condições de fertilidade de solos degradados.

Considerando a necessidade de revegetar áreas onde o solo foi extraído, razão por que adquiriu características químicas e biológicas distantes das ideais, este trabalho objetivou avaliar o uso de resíduos orgânicos como recondicionante de subsolo degradado e quantificar seus efeitos sobre a atividade microbiana e a fertilidade quando cultivado com barbatimão. 


\section{MATERIAL E MÉTODOS}

O experimento foi instalado em março de 2005 e conduzido até abril de 2006, em área degradada, localizada na Fazenda Experimental de Pesquisa e Extensão-FEPE, UNESP - Universidade Estadual Paulista, da Faculdade de Engenharia de Ilha Solteira, Município de Selvíria, MS. As coordenadas geográficas são $51^{\circ} 24^{\prime}$ WGr de longitude e $20^{\circ} 22^{\prime}$ S de latitude, com média anual de precipitação de $1.370 \mathrm{~mm}$. A temperatura média anual é de $24,7^{\circ} \mathrm{C}$, sendo janeiro e fevereiro os meses mais quentes (média de $26,3^{\circ} \mathrm{C}$ ) e junho e julho os mais frios (média de $21,5^{\circ} \mathrm{C}$ ). A umidade relativa média é de $67,9 \%$ (UNESP, 2006), e o clima predominante na região é do tipo Aw. O solo de maior ocorrência no local foi classificado como Latossolo Vermelho Distrófico (DEMATTÊ, 1980; EMBRAPA, 1999; MONIZ e CARVALHO, 1973).

Originalmente, a área apresentava cobertura vegetal tipo Cerrado sensu stricto. No final da década de 1960, com a construção da Usina Hidrelétrica de Ilha Solteira o local foi desmatado e parte do solo, retirada em profundidades de corte que variaram de 8 a $12 \mathrm{~m}$. Atualmente, nesse local, pequenas áreas estão em processo de regeneração natural, mas em sua maior extensão o subsolo permanece exposto, desprovido de cobertura vegetal e com a presença de acentuado processo erosivo (RODRIGUES et al., 2007).

As características químicas do subsolo no local foram analisadas antes da instalação do experimento. Uma amostra composta por quatro amostras simples, coletadas na profundidade de $0-0,15 \mathrm{~m}$, preparadas e analisadas segundo metodologia de Raij e Quaggio (1983), apresentou os seguintes resultados: $\mathrm{pH}\left(\mathrm{CaCl}_{2}\right)$ $=4,2 ; \mathrm{P}\left(\mathrm{mg}_{\mathrm{dm}}^{-3}\right)=1 ; \mathrm{MO}, \mathrm{K}, \mathrm{Ca}, \mathrm{Mg}, \mathrm{H}+\mathrm{Al}, \mathrm{Al}, \mathrm{SB}$, CTC, em mmol $\mathrm{dm}^{-3}=7 ; 0,3 ; 1 ; 1 ; 31 ; 9 ; 2,3$; e 33,3, respectivamente; e $\mathrm{V}(\%)=7$.

O aguapé utilizado foi coletado manualmente, com o auxílio de um gancho, em uma lagoa em área particular, próxima ao perímetro urbano de Ilha Solteira, SP. O bagaço de cana-de-açúcar foi doado pela Usina Pioneiros, localizada no Município de Sud Mennucci, SP. Aguapé e bagaço de cana foram secos ao ar e triturados para redução e uniformização do tamanho (cerca de $1 \mathrm{~cm}$ ). Uma amostra de cada material foi enviada para determinação dos teores de nutrientes, segundo Malavolta et al. (1997), cujos resultados seguem apresentados no Quadro 1.
A espécie arbórea utilizada como indicadora foi o barbatimão, cujas mudas foram produzidas a partir de sementes coletadas na região. As sementes foram escarificadas quimicamente, com ácido sulfúrico concentrado durante $20 \mathrm{~min}$, lavadas em água corrente, secas e recobertas com uma fina camada de $\mathrm{CaCO}_{3}$ e germinadas em papel germiteste. As plântulas foram transferidas para sacos plásticos de polipropileno contendo $2 \mathrm{~kg}$ de subsolo, o qual foi coletado na área do experimento, na profundidade de $0-0,15 \mathrm{~m}$, tendo corrigida a acidez e recebido adubação com $\mathrm{N}$ e $\mathrm{P}$, antes da introdução das mudas, que receberam rega diária em viveiro coberto. As mudas foram levadas para o lado de fora do viveiro aos 50 dias, onde permaneceram 10 dias, para adaptação às condições de campo, momento em que apresentaram em média $5 \mathrm{~cm}$ de altura.

Como condição básica para instalação do experimento, a área foi escarificada a $0,40 \mathrm{~m}$ de profundidade e gradeada. As covas $(0,30 \mathrm{~m}$ de diâmetro por $0,90 \mathrm{~m}$ de profundidade) foram abertas com emprego de broca hidráulica, no espaçamento 2 × $2 \mathrm{~m}$. O volume do subsolo da metade inferior da cova foi devolvido a esta, e os resíduos orgânicos foram misturados à metade do volume superior do subsolo de cada cova (cerca de $0,064 \mathrm{~m}^{3}$ ), na quantidade de $954 \mathrm{~g}$ de aguapé cova $^{-1}$, equivalente a 30 tha $^{-1}$ (SCHIAVETO et al., 2003), conforme os tratamentos, além da calagem ( $\left.15,9 \mathrm{~g} \mathrm{cova}^{-1}\right)$, adubação nitrogenada (12 $\left.\mathrm{g} \mathrm{cova}^{-1}\right)$ e fosfatada $\left(123,7 \mathrm{~g} \mathrm{cova}^{-1}\right)$, como se segue: TEST: testemunha; $\mathrm{C}$ : calagem; NP: adubação com $\mathrm{N}+\mathrm{P}$; CNP: calagem + N + P; NPA: $\mathrm{N}$ $+\mathrm{P}+$ aguapé; $\mathrm{NPB}: \mathrm{N}+\mathrm{P}+$ bagaço de cana; $\mathrm{NPAB}: \mathrm{N}$ $+\mathrm{P}+$ aguapé + bagaço de cana; $\mathrm{CNPA}$ : calagem $+\mathrm{N}+$ $\mathrm{P}+$ aguapé; $\mathrm{CNPB}$ : calagem $+\mathrm{N}+\mathrm{P}+$ bagaço de cana; e CNPAB: calagem $+\mathrm{N}+\mathrm{P}+$ aguapé + bagaço de cana.

No plantio das mudas foi depositado na cova a $0,10 \mathrm{~m}$ de profundidade, $50 \mathrm{~g}$ de solo inócuo, proveniente de uma área de Cerrado preservado da FEPE. Essa prática teve como objetivo introduzir ou aumentar a população de espécies de microrganismos, importante em qualquer processo de recuperação.

As mudas em campo foram irrigadas duas vezes por semana, utilizando-se um tanque-pipa, durante seis meses, até outubro, período coincidente com o início das chuvas. Para facilitar a retenção de água, foram feitos coroamentos nas covas, com raio de $0,60 \mathrm{~m}$, antes do plantio das mudas. Capinas foram realizadas aos 60 dias após o plantio e com intervalos de 30 dias, no perímetro da coroa e entre linhas.

R. Árvore, Viçosa-MG, v.32, n.6, p.1069-1079, 2008 
Quadro 1 - Análise química do aguapé e bagaço de cana-de-açúcar Table 1 - Chemical analysis of water hyacinth and sugar-cane bagasse

\begin{tabular}{|c|c|c|c|c|c|c|c|c|c|c|c|}
\hline & $\mathrm{N}$ & $\mathrm{P}$ & $\mathrm{K}$ & $\mathrm{Ca}$ & $\mathrm{Mg}$ & $\mathrm{S}$ & $\mathrm{B}$ & $\mathrm{Cu}$ & $\mathrm{Fe}$ & $\mathrm{Mn}$ & $\mathrm{Zn}$ \\
\hline & & & $\mathrm{g} \mathrm{kg}^{-1}$ & & & & \multicolumn{5}{|c|}{$\mathrm{mg} \mathrm{kg}^{-1}$} \\
\hline$\overline{\text { Aguapé }}$ & 8,61 & 0,83 & 8,45 & 25,15 & 6,08 & 3,43 & 43,15 & 18,30 & 1371 & 1148 & 9,50 \\
\hline Bagaço de cana & 3,92 & 0,09 & 1,18 & 1,18 & 2,10 & 0,55 & 11,06 & 33,40 & 900 & 104 & 41,20 \\
\hline
\end{tabular}

Durante a condução do experimento houve cinco períodos de amostragem de subsolo (junho, agosto, novembroe dezembrode 2005 e marçode 2006), emintervalos de aproximadamente 60 dias. As amostras de subsolo foram compostas a partir de cinco amostras simples, oriundas de covas escolhidas aleatoriamente, por tratamento e por repetição, à profundidade de 0 - $0,10 \mathrm{~m}$, utilizandose um trado de cilindro. Essas amostras foram secas ao ar, peneiradas, homogeneizadas e analisadas.

Parte dessas amostras foi enviada ao laboratório para análise química para fins de fertilidade, como anteriormente descrito, e a outra parte foi utilizada para análises microbiológicas, como se segue: carbono da biomassa microbiana (CBM), avaliado pelo método de fumigação-extração (VANCE et al., 1987); quantificação do carbono do $\mathrm{CO}_{2}\left(\mathrm{C}-\mathrm{CO}_{2}\right)$ liberado, segundo metodologia proposta por Anderson e Domsch (1982); quociente metabólico $\left(q \mathrm{CO}_{2}\right)$, determinado pela razão $\mathrm{C}-\mathrm{CO}_{2}$ liberado: biomassa microbiana, conforme Anderson e Domsch (1993) e quociente microbiano ( $q \mathrm{MIC}$ ), determinado pela relação $(\mathrm{MO} / 1,724) / \mathrm{MO} * 100$.

O delineamento utilizado foi o em blocos casualizados, com quatro repetições, ocupando cada um deles uma área de $250 \mathrm{~m}^{2}(10$ x $25 \mathrm{~m})$. Na análise dos dados, considerou-se o modelo de parcelas subdivididas em 10 tratamentos como parcelas e os cinco períodos de amostragens como subparcelas. Segundo o modelo adotado e em cada uma das variáveis, realizaram-se a análise de variância (Teste F) e suas complementações por meio do teste de Scott-Knott, nas comparações de médias de tratamentos e no estudo de regressão na análise de tendência ao longo das épocas de amostragens, ademais de alguns coeficientes de correlação linear. Nas análises foram utilizados os Programas SISVAR (FERREIRA, 1999-2003) e Statistical Analysis System (SAS, 1999).

\section{RESULTADOS E DISCUSSÃO}

Quanto à fertilidade do subsolo um ano após a aplicação dos tratamentos (Quadro 2), observaramse diferenças estatísticas entre os tratamentos e épocas de amostragem, com relação às variáveis analisadas. No Al houve interação significativa entre tratamento e amostragem de subsolo (Quadro 3). De maneira geral, o subsolo apresentou caráter ácido e pobre em nutrientes, podendo ser considerado degradado (MALAVOLTA e KLIEMANN, 1985), mesmo submetido a diferentes tratamentos (Quadro 2). Entretanto, verificaram-se sensível aumento nos valores de $\mathrm{P}, \mathrm{K}, \mathrm{Ca}, \mathrm{Mg}, \mathrm{SB}$, CTC e V e redução nos teores de $\mathrm{MO},(\mathrm{H}+\mathrm{Al})$ e $\mathrm{Al}$, em comparação com os anteriores à instalação do experimento.

Os maiores valores de $\mathrm{P}$ foram detectados no tratamento CNPA, seguido do CNP e CNPAB, mas, durante as épocas de amostragem, foram exibidos aumentos crescentes dos valores. Os benefícios foram proporcionados pelos resíduos vegetais, em razão da presença de microrganismos, que são responsáveis pela ciclagem de nutrientes, regulagem das transformações da matéria orgânica e disponibilização de elementos nutricionais, como o P (TURCO et al., 1994). Incrementos em $P$ já foram relatados em solo que receberam lodo de esgoto como resíduo orgânico (SILVA et al., 2008).

A variável MO apresentou diferenças entre os tratamentos que receberam e os que não receberam resíduos orgânicos (Quadro 2), sendo possível inferir sobre a contribuição dos resíduos utilizados em elevar o teor desta no subsolo. Ao longo do experimento, no entanto, observou-se desempenho decrescente dos valores de MO. Constatações como essas já foram relatadas, em que, com a adição de resíduos orgânicos, eram esperados incrementos nos teores de MO, o que não aconteceu de modo satisfatório, possivelmente devido ao fato de os resíduos terem sido aplicados em pequenas quantidades ou de apresentarem efeitos efêmeros, isto é, a MO foi mineralizada ao longo do experimento (SILVA et al., 2008). 
Quadro 2 - Médias, teste F (tratamentos, épocas de amostragem, interação tratamentos x coletas) e coeficientes de variação (CV\%) das características químicas do subsolo, coletado na profundidade de 0 - 0,10 m. Ilha Solteira, 2005/2006 Table 2 -Means, F Test (treatments, sampling times, interaction treatments $x$ sampling collections) and variation coefficients (VC\%) for the chemical characteristics of the subsoil, collected at the depth of O-0.10 m. Ilha Solteira, 2005/06

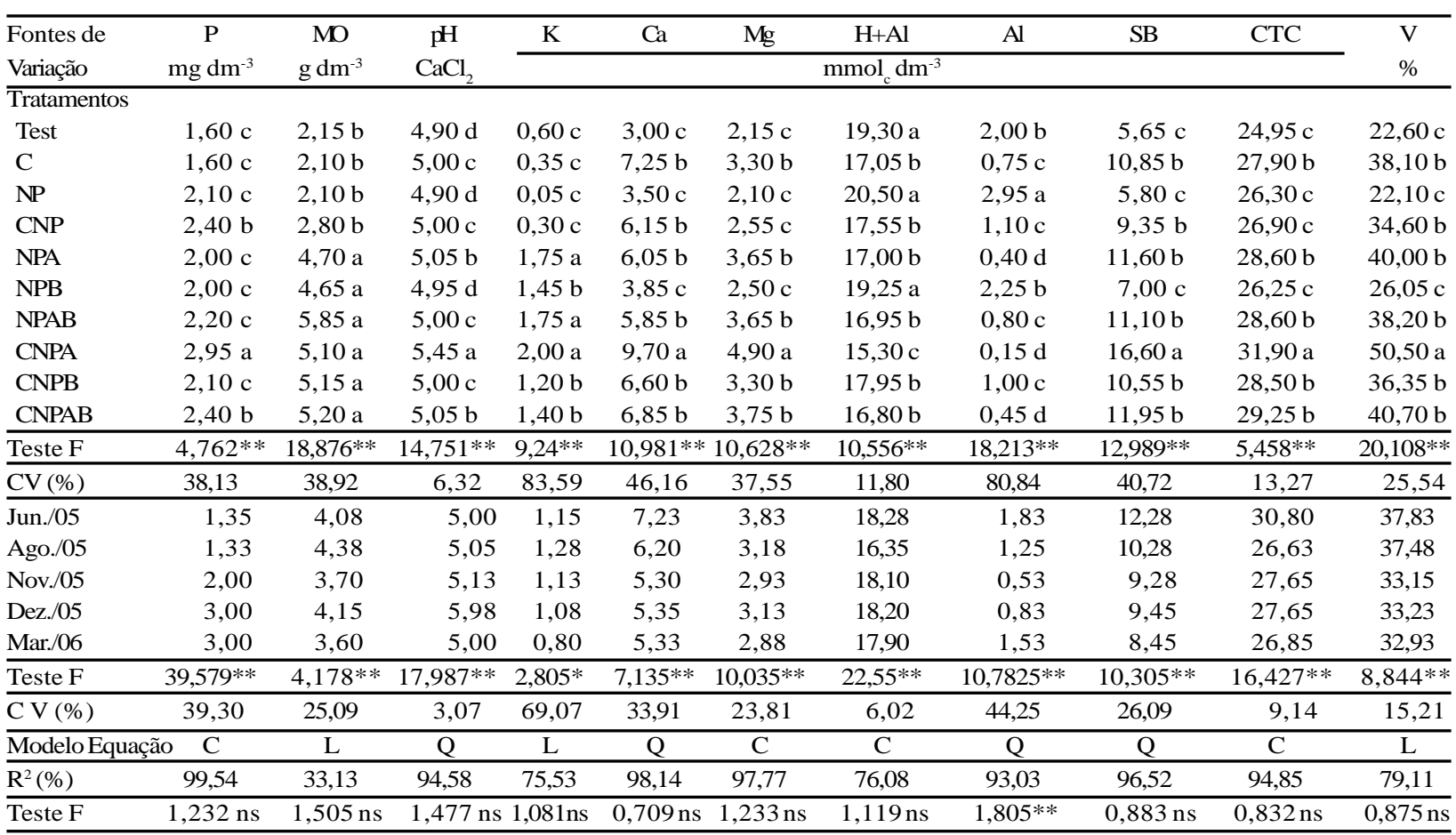

Médias seguidas de mesma letra, nas colunas, não diferem significativamente pela análise de Scott-Knott a 1\% de probabilidade. Tratamentos: Test: testemunha, C: calagem, NP: adubação com N + P, CNP: calagem + N + P, NPA: N + P + aguapé, NPB: N + P + bagaço de cana, NPAB: $\mathrm{N}+\mathrm{P}+$ aguapé + bagaço de cana, CNPA: calagem + N + P + aguapé, CNPB: calagem + N + P + bagaço de cana e CNPAB: calagem $+\mathrm{N}+\mathrm{P}+$ aguapé + bagaço de cana. ** e * significativos a 1 e 5\%, respectivamente; ns: não-significativo. L: Linear; Q: Quadrática e C: Cúbica.

Quanto ao $\mathrm{pH}$, constataram-se diferenças significativas entre os tratamentos (Quadro 2), sendo CNPA, NPA e CNPAB os tratamentos que possuíam em comum o maior valor de $\mathrm{pH}$, adubação com $\mathrm{N}$ e $\mathrm{P}$ e o aguapé. Ao longo do experimento, os valores de $\mathrm{pH}$ se processaram de maneira quadrática, e no final, os valores estavam diminuindo novamente. Tal fato pode ser explicado pela influência da calagem aplicada aos tratamentos. No entanto, considerando a condição da área, um subsolo, associado às baixas quantidades de bases trocáveis e ao caráter ácido, possivelmente fez que o efeito da calagem tivesse curta duração. Condição semelhante foi relatada num estudo de solos degradados, cujas características químicas do solo exibiram, também, baixos teores de $\mathrm{MO}$ e pH (SANTOS, 2004).

Os maiores valores de $\mathrm{K}$ foram proporcionados pelos tratamentos NPA, NPAB e CNPA, os quais possuíam em comum a adição de resíduos orgânicos (aguapé e, ou, bagaço de cana) e adubação com N + $\mathrm{P}$ (Quadro 2). Ao longo das amostragens, o K exibiu ajuste linear decrescente.

O Ca e o Mg mostraram desempenho semelhante; exibiram altos valores no tratamento CNPA e menores nos tratamentos TEST, NP e NPAB (Quadro 2). Isso pode ser explicado pela ação da calagem e associado ou não aos resíduos orgânicos. Maiores incrementos em Ca também foram observados por Silva et al. (2005), quando adicionaram aguapé ao solo. Ao longo deste trabalho, constatou-se desempenho quadrático e cúbico, respectivamente. Em um estudo dos efeitos da rochagem e da adubação orgânica sobre o crescimento de uma espécie arbórea do Cerrado, esses autores constataram maiores valores de $\mathrm{Ca}$ nos tratamentos que receberam aguapé seco e triturado (SILVA et al., 2008).

R. Árvore, Viçosa-MG, v.32, n.6, p.1069-1079, 2008 
Quadro 3 - Desdobramento da interação significativa dos tratamentos (Trat.) para $\mathrm{Al}\left(\mathrm{mmol}_{\mathrm{c}} \mathrm{dm}^{-3}\right)$ em diferentes épocas de amostragens de subsolo (EAS), coletadas na profundidade de $0-0,10 \mathrm{~m}$ e respectivos modelos de equação de regressão, $\mathrm{R}^{2}$ e significância dos valores de F. Ilha Solteira, 2005/2006

Table 3 - Unfolding of the significant interaction of the treatments (Treat.) for Al $\left(\mathrm{mmol}_{c} \mathrm{dm}^{-3}\right)$ at different times of subsoil samplings $(E A S)$, collected at the depth of $O-0.10 \mathrm{~m}$ and respective regression equation, $R^{2}$ and significance of the $F$ values for Ilha Solteira, 2005/06

\begin{tabular}{|c|c|c|c|c|c|c|c|}
\hline \multirow[t]{2}{*}{ Trat. } & \multicolumn{5}{|c|}{ Épocas de amostragens de subsolo } & \multirow[t]{2}{*}{ Equação } & \multirow{2}{*}{$\frac{\mathrm{R}^{2}}{(\%)}$} \\
\hline & Jun/05 & Ago/05 & Nov/05 & Dez/05 & Mar/06 & & \\
\hline Test & $2,75 \mathrm{~b}$ & $2,25 \mathrm{~b}$ & $1,25 \mathrm{a}$ & $2,00 \mathrm{a}$ & $1,75 \mathrm{~b}$ & $Y=3,8-1,189286 x+0,160714 x^{2} *$ & 69,43 \\
\hline $\mathrm{C}$ & $1,50 \mathrm{c}$ & $0,50 \mathrm{c}$ & $0,25 \mathrm{~b}$ & $0,75 \mathrm{~b}$ & $0,75 \mathrm{c}$ & $\mathrm{Y}=2,5-1,303571 \mathrm{x}+0,196429 \mathrm{x}^{2} * *$ & 79,59 \\
\hline NP & 3,75 a & 3,75 a & $1,50 \mathrm{a}$ & 2,25 a & $3,50 \mathrm{a}$ & $Y=2,45+2,851190 x-1,669643 x^{2}+0,229167 x^{3} * *$ & 79,45 \\
\hline CNP & $2,25 \mathrm{~b}$ & $1,00 \mathrm{c}$ & $0,50 \mathrm{~b}$ & $0,50 \mathrm{~b}$ & $1,25 \mathrm{~b}$ & $\mathrm{Y}=4,1-2,178571 \mathrm{x}+0,321429 \mathrm{x}^{2} * *$ & 99,83 \\
\hline NPA & $0,75 \mathrm{c}$ & $0,00 \mathrm{~d}$ & $0,00 \mathrm{~b}$ & $0,00 \mathrm{~b}$ & $1,25 \mathrm{~b}$ & $Y=2,1-1,614286 x+0,285714 x^{2} * *$ & 93,80 \\
\hline NPB & $3,25 \mathrm{a}$ & $2,25 \mathrm{~b}$ & $1,25 \mathrm{a}$ & $1,75 \mathrm{a}$ & $2,75 \mathrm{a}$ & $Y=5,45-2,507143 x+0,392857 x^{2} * *$ & 95,43 \\
\hline NPAB & $1,25 \mathrm{c}$ & $1,00 \mathrm{c}$ & $0,00 \mathrm{~b}$ & $0,50 \mathrm{~b}$ & $1,25 \mathrm{~b}$ & $\mathrm{Y}=2,7-1,55 \mathrm{x}+0,25 \mathrm{x}^{2} * *$ & 76,60 \\
\hline CNPA & $0,50 \mathrm{c}$ & $0,00 \mathrm{~d}$ & $0,00 \mathrm{~b}$ & $0,00 \mathrm{~b}$ & $0,25 \mathrm{c}$ & ns & - \\
\hline CNPB & $1,50 \mathrm{c}$ & $1,00 \mathrm{c}$ & $0,50 \mathrm{~b}$ & $0,50 \mathrm{~b}$ & $1,50 \mathrm{~b}$ & $\mathrm{Y}=2,9-1,55 \mathrm{x}+0,25 \mathrm{x}^{2} * *$ & 90,00 \\
\hline CNPAB & $0,75 \mathrm{c}$ & $0,75 \mathrm{c}$ & $0,00 \mathrm{~b}$ & $0,00 \mathrm{~b}$ & $0,75 \mathrm{c}$ & $Y=1,8-1,039286 x+0,160714 x^{2} *$ & 61,90 \\
\hline $\mathrm{T} \times \mathrm{EAS}$ & $1,805 * *$ & & & & & & \\
\hline $\mathrm{CV}(\%)$ & 44,25 & & & & & & \\
\hline
\end{tabular}

Médias seguidas de mesma letra nas colunas não diferem significativamente pela análise de Scott-Knott a $1 \%$ de probabilidade. Tratamentos: Test: testemunha, C: calagem, NP: adubação com N + P, CNP: calagem + N + P, NPA: N + P + aguapé, NPB: N + P + bagaço de cana, NPAB: $\mathrm{N}+\mathrm{P}+$ aguapé + bagaço de cana, CNPA: calagem $+\mathrm{N}+\mathrm{P}+$ aguapé, CNPB: calagem $+\mathrm{N}+\mathrm{P}+\mathrm{bagaço} \mathrm{de} \mathrm{cana} \mathrm{e} \mathrm{CNPAB:}$ calagem $+\mathrm{N}+\mathrm{P}+$ aguapé + bagaço de cana. $* * \mathrm{e}^{*}$ significativos a 1 e $5 \%$, respectivamente; ns: não-significativo.

Quanto à acidez potencial (H+Al), os maiores valores foram observados nos tratamentos TEST, NP e NPB, que apresentam em comum ausência de calagem, enquanto o menor valor foi observado no tratamento CNPA. Pode-se afirmar que a calagem contribuiu para a redução da $(\mathrm{H}+\mathrm{Al})$, associado ou não à adição dos resíduos orgânicos, com exceção do tratamento NPBA. Um ajuste cúbico foi encontrado nessa variável ao longo das amostragens. $\mathrm{O} \mathrm{Al}$ apresentou diferenças estatísticas significativas, destacando-se o tratamento NP com maior valor (Quadro 2). Os tratamentos C, CNP, NPA, CNPA, CNPB e CNPAB foram os que mostraram os menores valores de Al, possivelmente justificados pela ação da calagem, com exceção do NPAB, e associado, também, à adição dos resíduos. A interação entre tratamentos e épocas de amostragem foi significativa (Quadro 3), com valores menores verificados nos tratamentos NPA, CNPA e CNPAB, os quais receberam calagem e resíduos orgânicos, no caso dos dois últimos. Esse resultado foi observado por outros autores que, com a adição de resíduos orgânicos, verificaram um decréscimo da saturação por Al e aumento da eficiência no aproveitamento dos nutrientes pelas plantas cultivadas (IGUE e PAVAN, 1984).

Com relação a SB, CTC e V\%, verificaram-se parâmetros semelhantes entre os tratamentos e ao longo das amostragens de subsolo (Quadro 2), os maiores valores foram encontrados no tratamento CNPA e os menores no TEST, NPe NPB, que não receberam calagem. Ao longo do tempo, ocorreu redução em SB, CTC e V\%.

A recomendação do V\% nas culturas de reflorestamento é de 50\% (RAIJ et al., 1997). Nos tratamentos em análise, os valores observados foram inferiores a 50\%, exceto em CNPA, que, no entanto, exibiu valores muito baixos para $\mathrm{P}$ e $\mathrm{Mg}$, médios para $\mathrm{K}, \mathrm{pH}$ e V\%, e altos para Ca, em comparação com o Boletim 100 (RAIJ et al., 1997), resultados que podem ser explicados pelos efeitos da calagem, como promotora da diminuição da acidez, insolubilização e complexação de elementos tóxicos, como o Al, aumento nos teores de Ca e Mg, disponibilização de P e efeitos favoráveis à microflora do subsolo, bem como pela ação do resíduo vegetal (aguapé).

A variável CBM mostrou diferenças estatísticas entre os tratamentos e na interação tratamentos e épocas de amostragem (Quadro 4). Os tratamentos que receberam resíduos orgânicos (NPA, NPAB, CNPA, CNPB e CNPAB) foram os que proporcionaram valores superiores ao longo das medições, em especial na quarta e quinta amostragens. A irrigação do experimento foi suprimida em outubro, quando do início das chuvas, possibilitando observar o efeito positivo da mudança climática ocorrida sobre o crescimento das plantas e a atividade microbiana 
(CBM), efeito também conhecido como influência sazonal, em virtude da elevação da temperatura e da pluviosidade (TATE et al., 1991), que atinge principalmente as camadas superficiais do solo, onde as oscilações de umidade e temperatura são maiores (CATTELAN e VIDOR, 1990).

Neste trabalho, o CBM exibiu correlações significativa e positiva com diâmetro de colo, altura de planta e MO $\left(0,4985^{* *}, 0,5411^{* *}\right.$ e $0,2713^{* *}$, respectivamente) e negativa com $\mathrm{Al}\left(-0,1998^{* *}\right)$, mostrando relação positiva e direta do CBM com o crescimento da planta e com as condições de fertilidade do subsolo.

$\mathrm{O}$ C-CO $\mathrm{CO}_{2}$ liberado (Quadro 5) apresentou diferenças significativas entre tratamentos e na interação. Entre os tratamentos que receberam resíduos orgânicos, o NPA foi o único que exibiu variação quadrática ao longo do tempo, apresentando o maior valor na última amostragem do subsolo. Todos os tratamentos exibiram tendência ao equilíbrio. Dessa forma, os que receberam resíduos orgânicos apresentaram valores de $\mathrm{C}-\mathrm{CO}_{2}$ liberados superiores aos demais, comprovando alta atividade microbiana.

Os valores de $\mathrm{C}-\mathrm{CO}_{2}$ liberado foram superiores em $52 \%$ nas áreas com resíduos vegetais, em comparação com as áreas sem os citados resíduos, resultados semelhantes aos relatados por Assis Júnior et al. (2003). Correlações significativa e positiva de $\mathrm{C}-\mathrm{CO}_{2}$ liberado com $\mathrm{pH}$ foram observadas neste estudo $\left(0,4077^{* *}\right)$ e também no de Perez et al. (2004), os quais sugeriram que essa relação pode servir como indicador de estresse ambiental e que a atividade seria a maneira indireta de avaliação do impacto ambiental.

Diferenças significativas entre tratamentos e na interação entre tratamentos e épocas de amostragem foram detectadas em $q \mathrm{CO}_{2}$ (Quadro 6). Entre tratamentos, somente na primeira época de amostragem (junho de 2005) foram observadas diferenças, destacando-se o tratamento CNPAB com maior valor. Correlações significativa e negativa foram obtidas entre $q \mathrm{CO}_{2}$ e $\mathrm{CBM}\left(-0,5410^{* *}\right)$ e positiva em $\mathrm{C}-\mathrm{CO}_{2}$ liberado $\left(0,4169^{* * *}\right)$. As altas taxas de $q \mathrm{CO}_{2}$, como observadas na primeira época de amostragem, foram também relatadas por outros autores, que afirmaram que pode significar, em curto prazo, liberação de nutrientes para as plantas (PARKIN et al., 1996) e distúrbio ou estresses ambientais (SILVA, 2006).

Quadro 4 - Desdobramento da interação significativa de carbono de biomassa microbiana (CBM) $\left(\mu \mathrm{g} \mathrm{Cg}^{-1}\right.$ solo seco) entre os tratamentos (Trat.) e as diferentes épocas de amostragens de subsolo (EAS), coletadas na profundidade de 0 - 0,10 $\mathrm{m}$ e respectivos modelos de equação de regressão, $\mathrm{R}^{2}$ e significância dos valores de F. Ilha Solteira, 2005/2006

Table 4 - Unfolding of the significant interaction of microbial biomass carbon $(M B C)\left(\mu g \mathrm{C}^{-1}\right.$ dry soil) between the treatments (Trat.) and the different times of subsoil samplings (EAS), collected at the depth of $0-0.10 \mathrm{~m}$ and respective regression equation, $R^{2}$ and significance of the F values for F. Ilha Solteira, 2005/06

\begin{tabular}{|c|c|c|c|c|c|c|c|}
\hline \multirow[t]{2}{*}{ Trat. } & \multicolumn{5}{|c|}{ Épocas de amostragens de subsolo } & \multirow[t]{2}{*}{ Equação } & \multirow{2}{*}{$\frac{\mathrm{R}^{2}}{(\%)}$} \\
\hline & Jun/05 & Ago/05 & Nov/05 & Dez/05 & Mar/06 & & \\
\hline Test & $77,50 \mathrm{~b}$ & $90,50 \mathrm{~b}$ & $112,50 \mathrm{~b}$ & $148,25 \mathrm{~b}$ & $161,00 \mathrm{~b}$ & $\mathrm{Y}=50,525+22,475 \mathrm{x} * *$ & 97,31 \\
\hline $\mathrm{C}$ & $68,00 \mathrm{~b}$ & $71,75 \mathrm{~b}$ & $60,50 \mathrm{~b}$ & $157,75 \mathrm{~b}$ & $180,25 \mathrm{~b}$ & $Y=87,5-31,521429 x+10,428571 x^{2} *$ & 86,78 \\
\hline NP & $76,50 \mathrm{~b}$ & $98,75 \mathrm{~b}$ & $110,50 \mathrm{~b}$ & $101,25 \mathrm{c}$ & $169,75 \mathrm{~b}$ & $\mathrm{Y}=54,65+18,90 \mathrm{x} * *$ & 73,10 \\
\hline CNP & $108,00 \mathrm{~b}$ & $95,50 \mathrm{~b}$ & $112,50 \mathrm{~b}$ & $115,25 \mathrm{c}$ & $168,00 \mathrm{~b}$ & $Y=148,65-49,346429 x+10553571 x^{2} *$ & 98,75 \\
\hline NPA & $113,00 \mathrm{~b}$ & $103,00 \mathrm{~b}$ & $98,50 \mathrm{~b}$ & $211,00 \mathrm{a}$ & 195,75 a & $Y=302-280,3512 x+107,545 x^{2}-11,10417 x^{3} *$ & 84,74 \\
\hline NPB & $102,00 \mathrm{~b}$ & $135,00 \mathrm{a}$ & $122,25 \mathrm{~b}$ & $166,75 \mathrm{~b}$ & $163,00 \mathrm{~b}$ & $\mathrm{Y}=91,675+15,375 \mathrm{x} *$ & 78,68 \\
\hline NPAB & $103,25 \mathrm{~b}$ & $109,50 \mathrm{~b}$ & $121,00 \mathrm{~b}$ & $232,00 \mathrm{a}$ & $192,25 \mathrm{a}$ & $Y=283,6-279,964286 x+117535714 x^{2}-13 x^{3} * *$ & 87,12 \\
\hline CNPA & $146,50 \mathrm{~b}$ & $120,00 \mathrm{a}$ & $151,00 \mathrm{a}$ & $189,75 \mathrm{a}$ & 208,25 a & $\mathrm{Y}=105,125+19,325 \mathrm{x} * *$ & 74,27 \\
\hline CNPB & $243,00 \mathrm{a}$ & 158,25 a & $151,00 \mathrm{a}$ & 236,75 a & $215,00 \mathrm{a}$ & $\begin{array}{c}Y=564,15-456,952381 \mathrm{x}+154,839286 \mathrm{x}^{2}- \\
15,458333 \mathrm{x}^{3} * *\end{array}$ & 91,28 \\
\hline CNPAB & $82,50 \mathrm{~b}$ & $145,50 \mathrm{a}$ & $164,00 \mathrm{a}$ & $258,00 \mathrm{a}$ & $238,00 \mathrm{a}$ & $\mathrm{Y}=50,5+42,4 \mathrm{x} * *$ & 87,90 \\
\hline $\mathrm{T} \times \mathrm{EAS}$ & $2,115 * *$ & & & & & & \\
\hline $\mathrm{CV}(\%)$ & 26,26 & & & & & & \\
\hline
\end{tabular}

Médias seguidas de mesma letra nas colunas não diferem significativamente pela análise de Scott-Knott a $1 \%$ de probabilidade. Tratamentos: Test: testemunha, $\mathrm{C}$ : calagem, NP: adubação com N + P, CNP: calagem + N + P, NPA: N + P + aguapé, NPB: N + P + bagaço de cana, NPAB: $\mathrm{N}+\mathrm{P}+$ aguapé + bagaço de cana, CNPA: calagem + N + P + aguapé, CNPB: calagem + N + P + bagaço de cana e CNPAB: calagem $+\mathrm{N}+\mathrm{P}+$ aguapé + bagaço de cana. $* *$ e $*$ significativos a 1 e $5 \%$, respectivamente. 
Quadro 5 - Desdobramento da interação significativa para carbono do $\mathrm{CO}_{2}$ liberado $\left(\mathrm{C}-\mathrm{CO}_{2}\right.$ liberado) $\left(\mu \mathrm{g} \mathrm{CO} \mathrm{C}_{2}\right.$ solo seco $\mathrm{dia}^{-1}$ ) entre os tratamentos (Trat.) e as diferentes épocas de amostragens de subsolo (EAS), coletadas na profundidade de 0 - 0,10 m com respectivos modelos de equação de regressão, $R^{2}$ e significância para os valores de $F$. Ilha Solteira, 2005/2006

Table 5 - Unfolding of the significant interaction for released $\mathrm{CO}_{2}$ carbon (released $\left.\mathrm{C}_{-} \mathrm{CO}_{2}\right)\left(\mu \mathrm{g} \mathrm{CO}_{2} \mathrm{~g}_{\text {dry soil day }}{ }^{-1}\right)$ between the treatments (Treat.) and the different times of subsoil samplings (EAS), collected at the depth of $0-0.10 \mathrm{~m}$ with respective regression equation, $R^{2}$ and significance of the F values for Ilha Solteira, 2005/06

\begin{tabular}{|c|c|c|c|c|c|c|c|}
\hline \multirow[t]{2}{*}{ Trat. } & \multicolumn{5}{|c|}{ Épocas de amostragens de subsolo } & \multirow[t]{2}{*}{ Equação } & \multirow{2}{*}{$\frac{\mathrm{R}^{2}}{(\%)}$} \\
\hline & Jun/05 & Ago/05 & Nov/05 & Dez/05 & Mar/06 & & \\
\hline Test & $4,31 \mathrm{e}$ & $4,72 \mathrm{e}$ & $4,30 \mathrm{~d}$ & $4,72 \mathrm{~d}$ & $4,90 \mathrm{~d}$ & ns & - \\
\hline $\mathrm{C}$ & $5,29 \mathrm{~d}$ & $5,52 \mathrm{e}$ & $4,54 \mathrm{~d}$ & $5,25 \mathrm{~d}$ & $5,13 \mathrm{~d}$ & ns & - \\
\hline NP & $3,80 \mathrm{e}$ & $4,81 \mathrm{e}$ & $4,74 \mathrm{~d}$ & $5,36 \mathrm{~d}$ & $5,55 \mathrm{~d}$ & $\mathrm{Y}=3,6395+0,404 \mathrm{x} * *$ & 87,81 \\
\hline $\mathrm{CNP}$ & $4,12 \mathrm{e}$ & $5,22 \mathrm{e}$ & $4,83 \mathrm{~d}$ & $6,05 \mathrm{c}$ & $6,65 \mathrm{c}$ & $\mathrm{Y}=3,61+0,588 \mathrm{x} * *$ & 87,22 \\
\hline NPA & $6,80 \mathrm{c}$ & $6,94 \mathrm{~d}$ & $5,70 \mathrm{c}$ & $8,85 \mathrm{~b}$ & $10,13 \mathrm{a}$ & $\mathrm{Y}=8,452-2,00225 \mathrm{x}+0,47625 \mathrm{x}^{2} * *$ & 83,44 \\
\hline NPB & $9,90 \mathrm{~b}$ & $9,74 \mathrm{~b}$ & $6,53 \mathrm{c}$ & $6,44 \mathrm{c}$ & $7,20 \mathrm{c}$ & $Y=7,5895+4,674286 x-2,570714 x^{2}+0,325 x^{3} * *$ & 91,42 \\
\hline NPAB & $9,79 \mathrm{~b}$ & $8,71 \mathrm{c}$ & $6,03 \mathrm{c}$ & $8,20 \mathrm{~b}$ & $4,70 \mathrm{~d}$ & $Y=16,4035-9,096607 x+3,060893 x^{2}-0,34 x^{3} * *$ & 75,98 \\
\hline CNPA & $9,99 \mathrm{~b}$ & $8,39 \mathrm{c}$ & 10,98 a & $9,30 \mathrm{~b}$ & $8,99 \mathrm{~b}$ & $Y=12,974-4,955298 x+2,003661 x^{2}-0,236042 x^{3} *$ & 28,42 \\
\hline CNPB & $12,65 \mathrm{a}$ & 11,57 a & $11,30 \mathrm{a}$ & $8,34 \mathrm{~b}$ & $8,67 \mathrm{~b}$ & $Y=10,4505+3,707857 x-1,852768 x^{2}+0,206875 x^{3} *$ & 91,16 \\
\hline CNPAB & $12,53 \mathrm{a}$ & $8,96 \mathrm{c}$ & $10,06 \mathrm{~b}$ & $10,93 \mathrm{a}$ & $8,85 \mathrm{~b}$ & $\mathrm{Y}=23,9315-16,711131 \mathrm{x}+5,913661 \mathrm{x}^{2}-0,635208 \mathrm{x}^{3} *$ & 99,27 \\
\hline $\mathrm{T} \times \mathrm{EAS}$ & $16,387 * *$ & & & & & & \\
\hline $\mathrm{CV}(\%)$ & 9,39 & & & & & & \\
\hline
\end{tabular}

Médias seguidas de mesma letra nas colunas não diferem significativamente pela análise de Scott-Knott a 1\% de probabilidade. Tratamentos: Test: testemunha, C: calagem, NP: adubação com N + P, CNP: calagem + N + P, NPA: N + P + aguapé, NPB: N + P + bagaço de cana; NPAB: $\mathrm{N}+\mathrm{P}+$ aguapé + bagaço de cana, CNPA: calagem + N + P + aguapé, CNPB: calagem + N + P + bagaço de cana e CNPAB: calagem $+\mathrm{N}+\mathrm{P}+$ aguapé + bagaço de cana. $* *$ e $*$ significativo a 1 e $5 \%$, respectivamente; ns: não-significativo.

Quadro 6 - Desdobramento da interação significativa para quociente metabólico $\left(q \mathrm{CO}_{2}\right)\left(\mu \mathrm{g} \mathrm{CO}_{2} \mathrm{~g}_{\text {solo }} \operatorname{seco} \mathrm{dia}^{-1} / \mu \mathrm{g} \mathrm{C}\right.$ $\mathrm{g}^{-1}$ solo seco) entre os tratamentos (Trat.) e as diferentes épocas de amostragens de subsolo (EAS), coletadas na profundidade de 0 - 0,10 m, com os respectivos modelos de equação de regressão, $R^{2}$ e significância para os valores de F. Ilha Solteira, 2005/2006

Table 6- Unfolding of the significant interaction for metabolic quotient $\left(q \mathrm{CO}_{2}\right)\left(\mu \mathrm{g} \mathrm{CO} \mathrm{C}_{2} \mathrm{~g}\right.$ dry soil day ${ }^{-1} / \mathrm{\mu g} \mathrm{C}^{-1}$ dry soil) between the treatments (Treat.) and the different times of subsoil samplings (EAS), collected at the depth of $0-0.10$ $m$, with respective regression equation, $R^{2}$ and significance of the F values for F. Ilha Solteira, 2005/06

\begin{tabular}{|c|c|c|c|c|c|c|c|}
\hline \multirow[t]{2}{*}{ Trat. } & \multicolumn{5}{|c|}{ Épocas de amostragens de subsolo } & \multirow[t]{2}{*}{ Equação } & \multirow{2}{*}{$\frac{\mathrm{R}^{2}}{(\%)}$} \\
\hline & Jun/05 & Ago/05 & Nov/05 & Dez/05 & Mar/06 & & \\
\hline Test & $0,057 \mathrm{~d}$ & 0,055 & 0,038 & 0,036 & 0,032 & $\mathrm{Y}=0.064813-0.007068 \mathrm{x} *$ & 90,69 \\
\hline $\mathrm{C}$ & $0,089 \mathrm{c}$ & 0,079 & 0,082 & 0,035 & 0,029 & $\mathrm{Y}=0,111582-0,016312 \mathrm{x} * *$ & 83,13 \\
\hline NP & $0,051 \mathrm{~d}$ & 0,054 & 0,045 & 0,054 & 0,034 & ns & - \\
\hline $\mathrm{CNP}$ & $0,041 \mathrm{~d}$ & 0,057 & 0,052 & 0,054 & 0,040 & ns & - \\
\hline NPA & $0,064 \mathrm{~d}$ & 0,072 & 0,060 & 0,042 & 0,053 & $\mathrm{~ns}$ & - \\
\hline NPB & $0,123 \mathrm{~b}$ & 0,073 & 0,054 & 0,040 & 0,047 & $\mathrm{Y}=0,122598-0.018488 \mathrm{x} *$ & 76,83 \\
\hline NPAB & $0,098 \mathrm{c}$ & 0,080 & 0,051 & 0,038 & 0,027 & $\mathrm{Y}=0,114080-0,018350 \mathrm{x} *$ & 96,96 \\
\hline CNPA & $0,074 \mathrm{c}$ & 0,071 & 0,074 & 0,049 & 0,045 & $\mathrm{Y}=0,086583-0,008028 \mathrm{x} *$ & 78,01 \\
\hline CNPB & $0,067 \mathrm{~d}$ & 0,075 & 0,076 & 0,036 & 0,041 & $\mathrm{Y}=0,086425-0,009175 \mathrm{x} *$ & 57,91 \\
\hline CNPAB & $0,163 \mathrm{a}$ & 0,062 & 0,062 & 0,045 & 0,037 & $Y=0,367085-0,279853 x+0,081030-0 x^{2}, 007667 x^{3} *$ & 97,07 \\
\hline $\mathrm{T} \times \mathrm{EAS}$ & $3,048 * *$ & & & & & & \\
\hline $\mathrm{CV}(\%)$ & 36,13 & & & & & & \\
\hline
\end{tabular}

Médias seguidas de mesma letra nas colunas não diferem significativamente pela análise de Scott-Knott a 1\% de probabilidade. Tratamentos: Test: testemunha, C: calagem, NP: adubação com N + P, CNP: calagem + N + P, NPA: N + P + aguapé, NPB: N + P + bagaço de cana, NPAB: $\mathrm{N}+\mathrm{P}+$ aguapé + bagaço de cana, CNPA: calagem + N + P + aguapé, CNPB: calagem + N + P + bagaço de cana e CNPAB: calagem $+\mathrm{N}+\mathrm{P}+$ aguapé + bagaço de cana. $* *$ e $*$ significativo a 1 e $5 \%$, respectivamente; ns: não-significativo. 
A partir da segunda época de amostragem, os valores do $q \mathrm{CO}_{2}$ diminuíram, tendendo à estabilização do sistema, apresentando padrões lineares e cúbicos (Quadro 6). Avaliando agroecossistemas durante 21 anos, Mader et al. (2002) atribuíram um $q \mathrm{CO}_{2}$ baixo a uma maior diversidade da comunidade microbiana e maior eficiência no uso da energia. Esse desempenho indica que o sistema entra em equilíbrio, otimizando os efeitos da atividade microbiana e liberando menos $\mathrm{CO}_{2}$ para a atmosfera (GAMA-RODRIGUES et al., 1997).

A interação tratamento e épocas de amostragem foi significativa para $q \mathrm{MIC}$ (Quadro 7), tendo diferenças estatísticas detectadas entre tratamentos apenas nas duas primeiras épocas de amostragem (junho e agosto de 2005). Ao longo do período, os padrões de comportamento dos tratamentos foram lineares, quadráticos e cúbicos. Os tratamentos que receberam resíduos vegetais apresentaram valores de $q \mathrm{MIC}$ superiores aos demais, e isso demonstra, segundo Silva (2006), maior assimilação de matéria orgânica pelos microrganismos, proporcionando, dessa forma, condições para um crescimento superior das plantas. Observaram-se correlações significativa e positiva de $\mathrm{C}-\mathrm{CO}_{2}$ liberado e negativa para $\mathrm{CBM}\left(0,33157^{* * *} \mathrm{e}-\right.$ $0,15492^{* *}$, respectivamente), mostrando relação do $q \mathrm{MIC}$ com a atividade microbiana.

Quadro 7 - Desdobramento da interação significativa para quociente microbiano ( $q \mathrm{MIC})(\%)$ dos tratamentos (Trat.) em diferentes épocas de amostragens de subsolo (EAS), coletadas na profundidade de $0-0,10 \mathrm{~m}$ e respectivos modelos de equação de regressão, R2 e significância para os valores de F. Ilha Solteira, 2005/06

Table 7 - Unfolding of the significant interaction for microbial quotient (qMIC) (\%) of the treatments (Treat.) at different times of subsoil samplings (EAS), collected at the depth of $O-0.10 \mathrm{~m}$ and respective regression equation, $R^{2}$ and significance of the F values for F. Ilha Solteira, 2005/06

\begin{tabular}{|c|c|c|c|c|c|c|c|}
\hline \multirow[t]{2}{*}{ Trat. } & \multicolumn{5}{|c|}{ Épocas de amostragens de subsolo } & \multirow[t]{2}{*}{ Equação } & \multirow{2}{*}{$\frac{\mathrm{R}^{2}}{(\%)}$} \\
\hline & Jun/05 & Ago/05 & Nov/05 & Dez/05 & Mar/06 & & \\
\hline 1 & $1,341 \mathrm{~b}$ & $1,861 \mathrm{~b}$ & 1,085 & 0,945 & 0,766 & ns & - \\
\hline 2 & $1,638 \mathrm{~b}$ & $1,820 \mathrm{~b}$ & 2,561 & 0,781 & 0,664 & $\mathrm{Y}=0,829430+1,038240 \mathrm{x}-0,222855 \mathrm{x}^{2} *$ & 64,45 \\
\hline 3 & $1,117 \mathrm{~b}$ & $1,553 \mathrm{~b}$ & 1,115 & 1,545 & 0,721 & $\mathrm{~ns}$ & - \\
\hline 4 & $1,300 \mathrm{~b}$ & $1,794 \mathrm{~b}$ & 1,744 & 1,769 & 0,982 & $\mathrm{~ns}$ & - \\
\hline 5 & $2,309 \mathrm{~b}$ & 3,330 a & 2,672 & 1,379 & 1,345 & $Y=-2,1149+6,5663 x-2,3995 x^{2}+0,245 x^{3} *$ & 99,64 \\
\hline 6 & 3,608 a & $2,044 \mathrm{~b}$ & 2,057 & 1,328 & 2,009 & $Y=5,257100-1,997365 x+0,267645 x^{2} * *$ & 89,80 \\
\hline 7 & 3,659 a & 3,492 a & 2,057 & 1,894 & 1,652 & $\mathrm{Y}=4,273725-0,561100 \mathrm{x} * *$ & 91,76 \\
\hline 8 & $2,387 \mathrm{~b}$ & 3,196 a & 1,958 & 1,677 & 0,959 & $\mathrm{Y}=3,348015-0,437615 \mathrm{x} * *$ & 69,29 \\
\hline 9 & $1,840 \mathrm{~b}$ & $2,068 \mathrm{~b}$ & 1,797 & 1,162 & 1,311 & ns & - \\
\hline 10 & $4,662 \mathrm{a}$ & $1,970 \mathrm{~b}$ & 1,817 & 1,300 & 1,050 & $\mathrm{Y}=6,788055-2,726649 \mathrm{x} 0,322891 \mathrm{x}^{2} * *$ & 91,70 \\
\hline \multicolumn{8}{|c|}{$\begin{array}{ll}\mathrm{T} \times \mathrm{EAS} & 2,707 * * \\
\end{array}$} \\
\hline $\mathrm{C} \mathrm{V}(\%)$ & 39,86 & & & & & & \\
\hline
\end{tabular}

Médias seguidas de mesma letra nas colunas não diferem significativamente pela análise de Scott-Knott a $1 \%$ de probabilidade. Tratamentos: Test: testemunha, C: calagem, NP: adubação com N + P, CNP: calagem + N + P, NPA: N + P + aguapé, NPB: N + P + bagaço de cana, NPAB: $\mathrm{N}+\mathrm{P}+$ aguapé + bagaço de cana, CNPA: calagem + N + P + aguapé, CNPB: calagem + N + P + bagaço de cana e CNPAB: calagem $+\mathrm{N}+\mathrm{P}+$ aguapé + bagaço de cana. $* * \mathrm{e}^{*}$ significativo a 1 e $5 \%$, respectivamente; ns: não-significativo.

\section{CONCLUSÕES}

O subsolo continua a apresentar caráter ácido e pobreza em nutrientes, após o primeiro ano de avaliação. O carbono da biomassa mostrou tendências à estabilização, enquanto o quociente metabólico e o quociente microbiano diminuíram no mesmo período.

No conjunto, embora longe do ideal, os tratamentos com resíduo orgânico apresentaram melhores resultados, com ligeiro diferencial positivo para a presença de aguapé.

O CBM permitiu a observação de variação sazonal.

\section{AGRADECIMENTOS}

Ao CNPq e ao consórcio UNESP/Santander, pela concessão da bolsa de estudose aporte financeiro.

\section{REFERÊNCIAS}

ANDERSON, T. H.; DOMSCH, K. H. Ratios of microbial biomass carbon to total organic carbon in arable soils. Soil Biology and

Biochemistry, v.21, n.4, p.471-479, 1982. 
ANDERSON, T. H.; DOMSCH, K. H. The metabolic quotiente for $\mathrm{CO}_{2}\left(q \mathrm{CO}_{2}\right)$ as a specific activity parameter to assess the effects of environmental conditions, such $\mathrm{pH}$, on the microbial biomass of forest soils. Soil Biology and Biochemistry, v.25, n.3, p.393-395, 1993.

ASSIS JUNIOR, S. L.; ZANUNCIO, J. C.; KASUYA, M. C. Atividade microbiana do solo em sistemas agroflorestais, monoculturas, mata natural e área desmatada. Revista Árvore, v.27, n.1, p.35-41, 2003.

BEYRUTH, Z. Aquatic macrophytes from a marginal pond at Embu-mirim river. Revista de Saúde Pública, v.26, n.4, p.272-282, 1992.

CATTELAN, A. J.; VIDOR, C. Flutuações na biomassa, atividade e população microbiana do solo, em função de variações ambientais.

Revista Brasileira de Ciência do Solo, v.14, n.2, p.133-142, 1990.

CATTELAN, A. J.; VIDOR, C. Sistemas de culturas e a população microbiana do solo. Revista Brasileira de Ciência do Solo, v.14, n.2, p.125-132, 1992.

D’ANDRÉA, A. F. et al. Atributos biológicos indicadores da qualidade do solo em sistemas de manejo na região do cerrado no sul do estado de Goiás. Revista Brasileira de Ciências do Solo, v.26, n.4, p.913-924, 2002.

D'ALTERIO, C. F. V.; VALCARCEL, R. Medidas fisico-biológicas de recuperação de áreas degradadas: "Avaliação das modificações edáficas e fitossociológicas". In: JORNADA DE INICIAÇÃO CIENTIFICA, 6., 1996, Seropédica. Resumos... Seropédica: Universidade Federal Rural do Rio de Janeiro, 1996. p.52.

DEMATTÊ, J. L. I. Levantamento detalhado dos solos do "Campus experimental de Ilha Solteira”. Piracicaba: Escola Superior Agrícola Luiz de Queiroz, 1980. 119p.

\section{EMPRESABRASILEIRADEPESQUISA} AGROPECUÁRIA-EMBRAPA. Centro Nacional de Pesquisa de Solos. Sistema brasileiro de classificação do solo. Rio de Janeiro: 1999. 412p.

FEIGL, B. J. et al. Soil microbial biomass in Amazonian soils: evaluation of methods and estimates of pool sizes. Soil Biology and Biochemistry, v.27, n.11, p.1467-1472, 1998.

R. Árvore, Viçosa-MG, v.32, n.6, p.1069-1079, 2008
FERREIRA, D.F. SISVAR. Lavras: Universidade Federal de Lavras, 1999-2003. (Versão 4.2).

GAMA-RODRIGUES, E. F.; GAMA-RODRIGUES, A. C.; BARROS, N. F. Biomassa microbiana de carbono e de nitrogênio de solos sob diferentes coberturas florestais. Revista Brasileira de Ciência do Solo, v.21, n.3, p.361-365, 1997.

IGUE, K.; PAVAN, M. A. Uso eficiente de adubos orgânicos. In: SIMPÓSIO SOBRE FERTILIZANTES NAAGRICULTURA BRASILEIRA, 1., 1984, Brasília. Anais... Brasília: Embrapa-DEP, 1984. p.338-418.

LORENZI, H. Árvores brasileiras. 4.ed. Nova Odessa: Instituto Plantarum, 2002. v.2. p.204.

LORENZI, H. Plantas daninhas do Brasil: terrestre, aquáticas, parasitas, tóxicas e medicinais. 2.ed. Nova Odessa: Plantarum, 1991.p.440.

LUTZENBERGER, J. Ecologia: do jardim ao poder. 1985. Disponível em: <http://www.fgaia.org.br/texts/taguape.html>. Acesso em: 23 set. 2004.

MADER, P. et al. Soil fertility and biodiversity in organic farming. Science, v.296, n.5573, p.4, 2002.

MALAVOLTA, E.; KLIEMANN, H. J. Desordens nutricionais no cerrado. Piracicaba: Associação Brasileira para Pesquisa da Potassa e do Fosfato, 1985. 136p.

MALAVOLTA, E.; VITTI, G. C.; OLIVEIRA, S. A. Avaliação do estado nutricional das plantas: princípios e aplicações. 2.ed. Piracicaba: Potafos, 1997. 319p.

MARCONDES, D. A. S.; TANAKA, R. H. Plantas aquáticas nos reservatórios das usinas hidrelétricas das CESP. In: CONGRESSO BRASILEIRO DA CIÊNCIA DAS PLANTAS DANINHAS; WORKSHOP DE PLANTAS DANINHAS AQUÁTICAS, 21., 1997, Caxambu. Anais... Caxambu: Sociedade Brasileira da Ciência das Plantas Daninhas, 1997. p.2-4.

MONIZ, A. C.; CARVAlHO, A. Seqüência de evolução de solos derivados do arenito Bauru e de rochas básicas da região noroeste do estado de São Paulo. Bragantia, v.32, n.17, p.309-333, 1973. 
MONTEIRO, M. T.; GAMA-RODRIGUES, E. F. Carbon, nitrogen, and microbial biomass activity in different litter structures of a natural forest. Revista Brasileira de Ciências do Solo, v.8, n.5, p.819-826, 2004.

PAPENDIK, R.; PARRA, J. F. Soil quality: The key to sustainable agriculture american. Journal of Alternative Agriculture, v.7, n.1/2, p.2-3, 1992.

PARANHOS, S. B. Cana de açúcar: cultivo e utilização. Campinas: Fundação Cargill, 1987. v.2. 856p.

PARKIN, T. B.; DORAN, J. W.; FRANCO-VIZCAÍNO, E. Field and laboratory tests of soil respiration. In: DORAN, J. W.; JONES, A., (Eds.). Methods for assessing soil quality. Madison: Soil Science Society of America, 1996. p.231-245.

PEREZ, K. S. S.; RAMOS, M. L. G.; McMANUS, C. Carbono da biomassa microbiana em solo cultivado com soja sob diferentes sistemas de manejo nos cerrados. Pesquisa Agropecuária Brasileira, v.39, n.6, p.567-573, 2004.

RAIJ, B. van.; QUAGGIO, J. A. Métodos de análises de solos para fins de fertilidade. Campinas: Instituto Agronômico de Campinas, 1983. 31p. (Boletim Técnico, 81).

RAIJ, B. van. et al. Recomendações de adubação e calagem para o estado de São Paulo. Campinas: Instituto Agronômico de Campinas, 1997. 285p. (Boletim Técnico, 100).

RODRIGUES, G. B.; MALTONI, K. L.;

CASSIOLATO, A. M. R. Dinâmica da regeneração do subsolo de áreas degradadas dentro do bioma Cerrado. Revista Brasileira de Engenharia Agrícola e Ambiental, v.11, n.1, p.73-80, 2007.

SANTOS, M. C. Carbono da biomassa microbiana, do $\mathrm{CO}_{2}$ liberado e micorrização em pastagens degradadas. 2004. 54f. Dissertação (Mestrado em Produção Vegetal) - Universidade Estadual Paulista, Ilha Solteira, 2004.

SAS Institute. - Statistical Analysis System Institute. SAS/STAT Procedure guide for personal computers. 9.ed. Cary: 1999. 334p.
SCHIAVETO, A. R.; MALTONI, K. L.;

CASSIOLATO, A. M. R. Utilização do aguapé como fonte de matéria orgânica e nutrientes na recuperação de áreas degradadas. In: CONGRESSO DE INICIAÇÃO CIENTÍFICA DA UNESP, 15., 2003, Marília. Anais... São Paulo/UNESP, 2003. CD ROM.

SILVA, M. S. C. Indicadores de qualidade do solo em sistemas agroflorestais em Paraty, RJ. 2006. 68p. Dissertação (Mestrado em Agronomia) - Universidade Federal Rural do Rio de Janeiro, Seropédica, 2006.

SILVA, E. A. et al. Efeitos da rochagem e de resíduos orgânicos sobre aspectos químicos e microbiológicos de um subsolo exposto e no crescimento de Astronium fraxinifolium Schott. Revista Árvore, v.32, n.2, p.323-333, 2008.

\section{SOUZA, V. C.; LORENZI, H. Botânica}

sistemática: guia ilustrado para identificação das famílias de Angiospermas da flora brasileira, baseado em APG II. Nova Odessa: Instituto Plantarum, 2005. 640p.

TATE, K. R. et al. Microbial biomass of measurement procedures, temporal variations, and the influence of $\mathrm{P}$ fertility status. Plant and Soil, v.132, n.2, p.233-241, 1991.

TURCO, R. F.; KENNEDY. A. C.; JAWSON, M. D. Microbial indicators of soil quality. In: DORAN. J. W. et al. (Eds.). Defining soil quality for a sustainable environment. Madison: Soil Science Society of America, 1994. p.73-90. (SSSA Special Publication, 35).

ULLER, A. Introdução. In: INSTITUTO CUBANO DE PESQUISA DOS DERIVADOS DA CANA-DEAÇÚCAR - INCIDCA. Manual dos derivados da cana-de-açúcar: diversificação, matérias primas, derivados do bagaço, derivados do melaço, outros derivados, resíduos, energia. Brasília: ABIPTI, 1999. 474p.

UNIVERSIDADE ESTADUAL PAULISTA - UNESP. Departamento de Fitossanidade, Engenharia Rural e Solos/Área de Hidráulica e Irrigação. Dados climáticos de Ilha Solteira. Disponível em: <http//www.agr.feis.unesp.br/clima.php>. Acesso em: 23 out. 2006.

VANCE, E. D.; BROOKES, P. C.; JENKINSON, D. $\mathrm{S}$. An extraction method for measuring soil microbial biomass C. Soil Biology and Biochemistry, v.19, n.6, p.773-777, 1987.

R. Árvore, Viçosa-MG, v.32, n.6, p.1069-1079, 2008 